\title{
The Carcinogenic Liver Fluke Opisthorchis viverrini among Rural Community People in Northeast Thailand: a Cross- Sectional Descriptive Study using Multistage Sampling Technique
}

\author{
Soraya J Kaewpitoon ${ }^{1,2,3 *}$, Natthawut Kaewpitoon ${ }^{1,2,4}$, Ratana Rujirakul ${ }^{1}$, Naporn \\ Ueng-arporn ${ }^{1,2,5}$, Likit Matrakool ${ }^{2,6}$, Taweesak Tongtawee ${ }^{2,6}$
}

\begin{abstract}
Opisthorchis viverrini infection is a serious public health problem in Southeast Asia especially in the northeast and north of Thailand. Therefore, a cross-sectional survey using multistage sampling was conducted from the rural communities of Surin province, Thailand, during September 2013 to July 2014. O. viverrini infection was determined using Kato's thick smear technique. Socio-demographic, information resources, and history data were collected using predesigned semi-structured questionnaires. A total of 510 participants completed interviews and had stools collected. Some $32(6.47 \%)$ participants were infected with $O$. viverrini. The rate was slightly higehr in males $(6.61 \%)$ than females $(6.32 \%)$. High frequencies were found in the age groups $61-70(19.4 \%)$ and $71-80$ years $(19.4 \%)$, those involved in agriculture $(10.5 \%)$, and in primary school $(10.3 \%)$. The distribution of high infection was found in Tha Tum (16.7\%) and Sankha district (16.7\%), followed by Samrong Thap (13.3\%), Si Narong (13.33\%), and Buachet district (13.33\%). Chi-square testing indicated that age (61-70 and 71-80 year old), education (primary school) and occupation (agriculture), were significantly associated with $O$. viverrini infection (p-value $<0.05$ ). Of $72.6 \%$ participants who had past histories with stool examination, $17.0 \%$ of them had been infected with $O$. viverrini and $43.2 \%$ treated with praziquantel. This finding confirmed that $O$. viverrini is still a problem in Surin province, Thailand, and therefore, interventions are urgently required for mass treatment and health education implementation.
\end{abstract}

Keywords: Carcinogenic human liver fluke - Opisthorchis viverrini - Surin - Thailand

Asian Pac J Cancer Prev, 16 (17), 7803-7807

\section{Introduction}

Opisthorchis viverrini, carcinogenic liver fluke, is an endemic in the Lower Mekong Basin, including Thailand, Lao People's Democratic Republic (Lao PDR), Cambodia and central Vietnam (Sripa et al., 2010). The under-estimate of infections are considered, more than 10 million people are infected with $O$. viverrini in Thailand and Lao PDR (Sripa et al., 2010; Sithithaworn et al., 2012). In Thailand, it is estimated that 6 million people are infected with the $O$. viverrini (Sithithaworn et al., 2012). This figure indicated that it is a serious public health problem in Thailand, particularly in northeastern and northern region (Kaewpitoon et al., 2008; Sripa et al., 2010; Sithithaworn et al., 2012). A community-level health education campaign been conducted since late 1950s. O. viverrini control has been started as a small scale helminthiasis control program in some high risk areas. A large scale has been started, the program is operated in some provinces of the central and all provinces of the northeast and north of Thailand. The main strategies for liver fluke control comprise three interrelated approaches, namely stool examination and treatment of positive cases with praziquantel for eliminating human host reservoir, health education for a promotion of cooked fish consumption to prevent infection, and improvement of hygienic defecation for the interruption of disease transmission (Jongsuksantikul and Imsomboon, 2003; Sithithaworn et al., 2012). The $O$. viverrini infection is associated with hepatobiliary diseases including hepatomegaly, cholangitis, cholecystitis, and gallstones (Harinasuta and Vajrasthira 1960; Harinasuta et al., 1984). Recently, O. viverrini has been classified as Type 1 carcinogens by the International Agency for Research on Cancer, World Health Organization (WHO) (Thamavit et al., 1978; Harinasuta et al., 1984). The O.viverrini infection in Thailand was the first reported in 1955 (Sadun) and many strategies has been operated over period 1955-

${ }^{1}$ Parasitic Disease Research Unit, Institute of Medicine, Suranaree University of Technology, ${ }^{2}$ Division of Research Clinic, Suranaree University of Technology Hospital, ${ }^{4}$ Faculty of Public Health, Vongchavalitkul University, ${ }^{3}$ Department of Family and Community Medicine, ${ }^{5}$ Department of Pediatric, ${ }^{6}$ Department of Surgery, Institute of Medicine, Suranaree University of Technology, Nakhon Ratchasima, Thailand*For correspondence: soraya.k@sut.ac.th 
2000 , the national prevalence of $O$. viverrini infection had fallen from $63.6 \%$ to $9.6 \%$ but the high prevalence rate is still found in the rural communities of provinces, Northeast (Sithithaworn et al., 2012). In addition, the high mortality rate of CCA was reported in the northeast areas where found frequently of $O$. viverrini infection (Sripa et al., 2010). Therefore, to prevent and control of CCA, the screening of $O$. viverrini infection is more required. This cross-sectional survey using multistage sampling was conducted from 17 districts of Surin province, Thailand. This research data could be used to reduce the morbidity and mortality rate of $O$. viverrini infection and CCA, respectively.

\section{Materials and Methods}

A cross-sectional survey was conducted in Surin province where is located in the northeast of Thailand, with 450 kilometers from Bangkok (capital) by road and 420 kilometers by train. The province is subdivided into 17 districts. The districts are further subdivided into $158 \mathrm{sub}-$ districts and 2,120 villages, 441,922 houses, and 1,381,761 populations (691,425 males and 690,226 females) (Surin Administrative Organization, Thailand 2013). A survey of $O$. viverrini infection in 17 districts of Surin province was carried out during September 2013 to July 2014.The participants were selected by multistage sampling with cluster size of 30 . The estimated sample size required was 510 and was calculated using the following formula:

$$
\begin{aligned}
& Z^{2}(\alpha / 2) N P_{(1-p)} x \text { design effect } \\
& Z^{2}(\alpha / 2) P_{(1-p)}+(N-1) d^{2}
\end{aligned}
$$

$\mathrm{N}=$ population $=1,391,635$

$\mathrm{Z}=1.9695 \%(\alpha=.05)$

Design effect: $\mathrm{df}=2$

$\mathrm{D}=$ acceptable margin of error $=0.05$

$\mathrm{P}=$ estimated prevalence rate $O$. viverrini infection $=0.161$ (Sithithaworn et al., 2012)

Multistage sampling was used to select the samples in this studied, cluster sampling; selected 17 sub-districts from 17 districts, and also the selection of 17 villages registered for each district. Simple random sampling was used to select the samples in the villages with cluster size of 30 (Figure 1). One participant over 4 years of age, who had been living in the sub-district for at least 6 months, was randomly chosen within each household at the time of visiting by a research assistant. Necessary permission from the concerned authorities was taken and a survey was conducted using semi-structured questionnaires included personal data (gender, age, occupation, and education), information resources regarding the $O$. viverrini prevention and control (human and media sources), the histories of stool examination, $O$. viverrini infection, and treatment, Prior informed consent was taken. For those not available in the first interview another visit was made to minimize non-response.

Stools were collected from 30 participants/each districts (who had completed the interviewed) and kept in labeled plastic bags and then transported in an ice box to the laboratory at the Parasitic Disease Research Unit, Department of Pathology, Institute of Medicine,
Suranaree University of Technology, Thailand, within a day after collection. Stool specimens were examined the $O$. viverrini and other known intestinal parasitic egg by the Kato thick smear procedures according to the method of Kato and Miura (1954). Briefly, the materials used were prepared in accordance with standard laboratory in-house procedures. Thus, the glycerin-malachite green solution was mixed with $1 \mathrm{ml}$ of $3 \%$ malachite green, $100 \mathrm{ml}$ of $6 \%$ phenol and $100 \mathrm{ml}$ of pure glycerin. The cellophane strips, each $22 \times 40 \mathrm{~mm}$, were soaked in this solution for at least 24 hours before use. Additionally, in order to eliminate fibers or seed, the technique was modified by pressing a 105-mesh stainless steel grid onto the sample which was then filtered, transferred to slides covered by the cellophane soaked cover slips and allowed to stand for 30 minutes. All preparations were initially screened with a low-power (10x) objective lens. Suspected parasitic

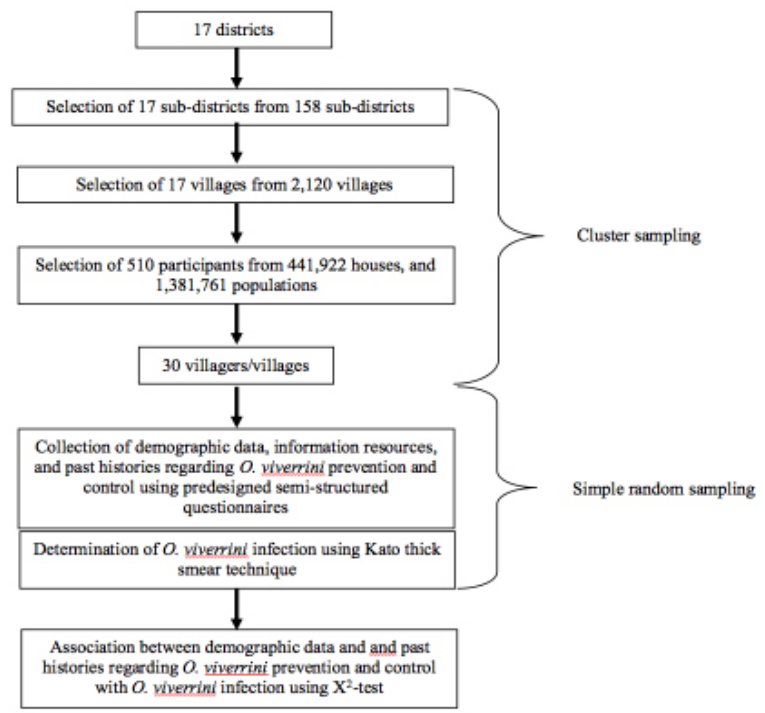

Figure 1. Flowchart of Study Design Regarding a Carcinogenic Liver Fluke $\boldsymbol{O}$. viverrini among Rural Community People in Surin Province, Northeast Thailand

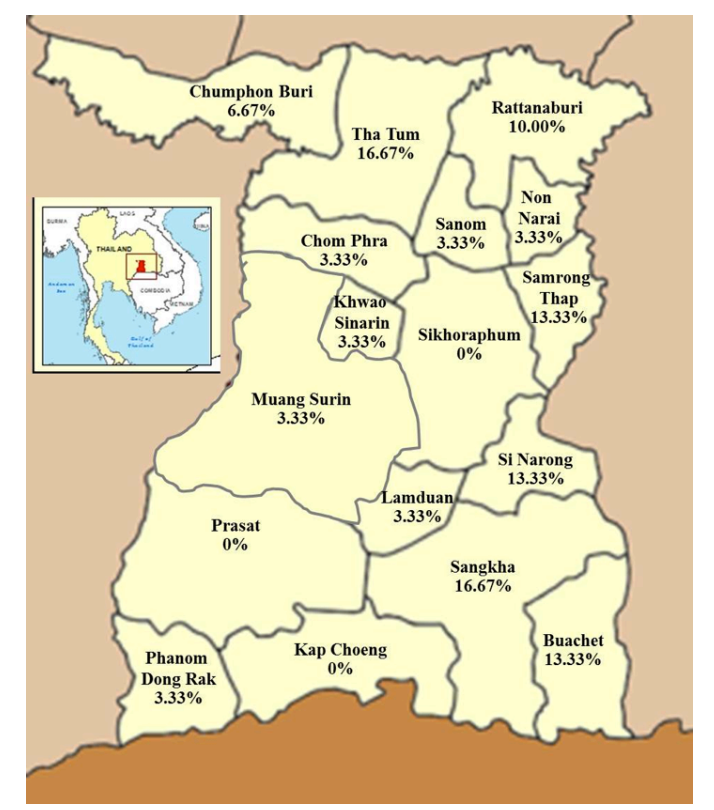

Figure 2. Distribution of $O$. viverrini Infection in Villagers from 17 Districts of Surin Province, Thailand 
Opisthorchis viverrini among Rural People in Northeast Thailand: a Cross-sectional Descriptive Study

objects were subsequently examined under a high-power (40x) objective. The stool samples were preserved in $10 \%$ formalin for later confirmation, if needed. O. viverrini positive case was confirmed by 2 expert parasitologists before a definitive diagnosis was established. Patients who infected with other known parasitic were treated with anti-parasitic drugs and also attended the health education. The data were analyzed with descriptive statistics, and chi-square tests were used to investigate the associations between demographic and histories data with $O$. viverrini infection. Statistical data analysis was carried out using SPSS software version 12.0. The study protocol was approved by Suranaree University Ethical Review Committee, EC55-57.

\section{Results}

Of $32(6.47 \%)$ from 510 eligible participants was infected with $O$. viverrini. The $O$. viverrini infection rate was males $(6.61 \%)$ and females $(6.32 \%)$. Most of the infected participants were found in the age group 61$70(19.40 \%)$ and $71-80$ year old (19.35\%), agriculture (10.51\%), and primary school level $(10.26 \%)$, respectively (Table 1). The highest of infection was found in Tha Tum (16.67\%), and Sankha district (16.67\%), followed by Samrong Thap (13.33\%), and Buachet district (13.33\%), respectively (Figure 2). Participants were completed the questionnaires about the information resources regarding

Table 1. O. viverrini Infection in 17 Villagers of Surin Province, Thailand by Gender, Age, Occupation, and Education

\begin{tabular}{|c|c|c|c|c|}
\hline Personal Data & $\begin{array}{l}\text { No. of } \\
\text { rticipants }\end{array}$ & $\begin{array}{c}\text { O. viverrini } \\
\text { infection }\end{array}$ & $\%$ & $95 \% \mathrm{CI}$ \\
\hline \multicolumn{5}{|l|}{ Sex } \\
\hline male & 242 & 16 & 6.61 & $0.10-0.21$ \\
\hline female & 268 & 17 & 6.32 & $0.11-0.30$ \\
\hline \multicolumn{5}{|l|}{ Age } \\
\hline $5-10$ & 40 & 0 & 0.00 & \\
\hline $11-20$ & 50 & 0 & 0.00 & \\
\hline $21-30$ & 56 & 1 & 1.79 & \\
\hline $31-40$ & 56 & 0 & 0.00 & \\
\hline $41-50$ & 58 & 1 & 1.72 & \\
\hline $51-60$ & 65 & 3 & 4.62 & \\
\hline $61-70$ & 67 & 13 & $19.40^{*}$ & $0.19-0.45$ \\
\hline $71-80$ & 62 & 12 & $19.35^{*}$ & $0.16-0.33$ \\
\hline $81-90$ & 56 & 3 & 5.36 & \\
\hline \multicolumn{5}{|l|}{ Occupation } \\
\hline Agriculture & 257 & 27 & $10.51 *$ & $0.12-0.31$ \\
\hline Employee & 76 & 2 & 2.63 & \\
\hline Student & 53 & 0 & 0.00 & \\
\hline Unemployable person & n 43 & 3 & 6.98 & \\
\hline Housewife & 32 & 1 & 3.13 & \\
\hline Trader & 28 & 0 & 0.00 & \\
\hline Government officer & 21 & 0 & 0.00 & \\
\hline \multicolumn{5}{|l|}{ Education } \\
\hline Primary school & 302 & 31 & $10.26^{*}$ & $0.11-0.29$ \\
\hline Secondary school & 128 & 1 & 0.78 & \\
\hline Unlearned & 30 & 1 & 3.33 & \\
\hline University & 21 & 0 & 0.00 & \\
\hline Vocational college & 29 & 0 & 0.00 & \\
\hline Total & 510 & 33 & 6.47 & $0.15-0.35$ \\
\hline
\end{tabular}

* statistical significant p-value $<0.05$
Table 2. Information Resources Regarding $O$. viverrini Prevention and Control in Surin Province, Thailand

\begin{tabular}{lcr}
\hline Information resources & No. of participants & $\%$ \\
\hline Human & & \\
Village health volunteer & 144 & 28.24 \\
Public health officer & 140 & 27.45 \\
Village headman & 137 & 26.86 \\
Nurse & 40 & 7.84 \\
Medical doctor & 20 & 3.92 \\
Teacher & 18 & 3.53
\end{tabular}

Chief executive of the sub-district administrative organization

\begin{tabular}{lrr} 
Media & & \\
Television & 214 & 41.96 \\
Village news station & 109 & 21.37 \\
Pamphlet & 105 & 20.59 \\
Newspaper & 51 & 10.00 \\
Internet & 20 & 3.92 \\
Poster & 11 & 2.16 \\
\hline
\end{tabular}

Table 3. The Past Histories with Stool Examination, $O$. viverrini Infection, and Praziquantel used, in Surin Province, Thailand

\begin{tabular}{llcc}
\hline Past histories & & No. of participants & $\%$ \\
\hline Stool Examination & Yes & 370 & 72.55 \\
& No & 140 & 27.45 \\
O. viverrini infection & Yes & 98 & 17.04 \\
& No & 477 & 82.96 \\
Praziquantel used & Yes & 294 & 43.24 \\
& No & 386 & 56.76 \\
\hline
\end{tabular}

$O$. viverrini prevention and control. $28.24 \%$ of village health volunteer was the main human source, followed by $27.45 \%$ of public health officer, and $26.86 \%$ of and village headman, were the main information resources regarding $O$. viverrini prevention and control. $41.96 \%$ of television was the main media source, followed by $21.37 \%$ and $20.59 \%$ of village news station and pamphlet, were the main information resources regarding $O$. viverrini prevention and control (Table 2). The past histories with stool examination, $O$. viverrini infection, and praziquantel used, were interviewed. Of $72.55 \%$ participants had past histories with stool examination, $17.04 \%$ of them had infected with $O$. viverrini, $43.24 \%$ participants had treated with praziquantel (Table 3). Chi-square testing indicated that age groups were significantly associated with $O$. viverrini infection $(\mathrm{p}$-value $<0.05)$. The prevalence of $O$. viverrini infection increase with age, and statistically significant with age group $61-70$ year old, $\mathrm{X}^{2}(\mathrm{~N}=67)=10.31$, $\mathrm{p}<0.05$, and 71-80 year old, $\mathrm{X}^{2}(\mathrm{~N}=62)=9.29, \mathrm{p}<0.05$. The prevalence of $O$. viverrini infection increase with education and occupation, and statistically significant with primary school level, $\mathrm{X}^{2}(\mathrm{~N}=302)=8.49, \mathrm{p}<0.05$, and agriculture, $\mathrm{X}^{2}(\mathrm{~N}=257)=8.13, \mathrm{p}<0.05$. The proportion of $O$. viverrini infected was slightly higher in males than in females, but the gender different was not statistically significant, $\mathrm{X}^{2}(\mathrm{~N}=510)=1.10, \mathrm{p}=0.13$.

\section{Discussion}

Opisthorchiasis caused by $O$. viverrini, is of considerable public health importance in Southeast Asia, 
particularly in Lao PDR and Thailand (Sripa et al., 2010). Human have been infected by ingesting under-cooked fish containing infective metacercariae, this is very common in the northeastern and northern region particularly in rural areas (Sadun 1995; Wykoff et al., 1966; Vichasri et al., 1982; Sithithaworn et al., 1997; Jongsuksantikul and Imsomboon 2003). Although, many program campaign has been operated in Thailand for reduced $O$. viverrini infection over period 1955-2000, the national prevalence of $O$. viverrini infection had fallen but the high prevalence rate is still found in the rural communities of provinces, Northeast (Jongsuksantikul and Imsomboon 2003; Sithithaworn et al., 2012). Recent study, O. viverrini infection was $6.76 \%$, this is a first reported a provincewide survey in all age group in rural community of Surin province, Thailand. The highest prevalence was among those between age group 61-70 (19.40\%) and 71-80 year old $(19.35 \%)$; similar to other studied (Kaewpitoon et al., 2012b; Kaewpitoon et al., 2012c; Thaewnongiew et al., 2014). Previous studied in Surin province, $9.91 \%$ of elderly were infected with $O$. viverrini (Kaewpitoon et al., 2012c). However, this recent reported shows a higher than the past studied, a large-scale is conducted in this studied due to clear. The proportion of $O$. viverrini infected was slightly higher in males than in females, but the gender different was not statistically significant. People who had an education level with primary school and had an occupation concerning with agriculture, were statistically significantly associated with $O$. viverrini infection, similar to other studied (Thaewnongiew et al., 2014; Suwannahitatom et al., 2013). This figure shows that the middle age and elderly, primary school, and agriculture, are under the need for urgently introduce mass treatment and health education.

The distribution of $O$. viverrini in Surin province was found frequently in 13 from 17 districts, this result indicates that people are still eating fresh fishes containing $O$. viverrini infective stage. A highest prevalence of $O$. viverrini infection was found in Tha Tum, Sankha, Samrong Thap, Buachet and Si Narong district, this result similar to the other studied that had been localization of the risk areas for $O$. viverrini infection. By GIS analysis, Si Narong, Sangkha, Mueang Surin, Non Narai, Samrong Thap, Chumphon Buri, Tha Tum, Buachet, and Rattanaburi to have the highest risk areas in Surin province, Thailand (Rujirakul et al., 2015). A solution for infection prevention needs to be quickly investigated especially for middle-aged and elderly males. When people engage in less risky behaviors, the need and consumption of anti-anthelminthic treatment will be reduced. A health education campaign needs to be organized in areas of the top 5 districts. Present study shows that the village health volunteer, public health officer, and village headman were the important messengers for $O$. viverrini prevention and control. Therefore, the correct of knowledge, attitude, and practices regarding $O$. viverrini information are required. In addition, participants perceived the $O$. viverrini information through television, village news station, and pamphlet, this figure indicates that it may be a useful tool for rural community people improvement via 3 main media. The effectiveness of control programs and its use on a community basis was to prove a major step forward in subsequent control strategies, and region-wide control programs were established from 1987 onwards under the Five-year National Development Plan for 1987-1991. A concise history of opisthiorchiasis control programs in Thailand, and their apparent effectiveness has been detailed by Jongsuksuntigul and Imsomboon (2003) and Sripa et al. (2011). Present study, of $72.55 \%$ participants had a history with stool examined, $17.04 \%$ had past history with $O$. viverrini infection, and $43.24 \%$ had past history with praziquantel used, this result also shown that strategies for the control of $O$. viverrini infection still required for stool examination and treatment of infected cases. Past studied, multiple logistic regression analysis was the used to investigate $O$. viverrini infection factors in the target group, 3,916 Thai residents of northeast Thailand who were 15 or over. The remaining 6 variables were sex, age, water resource habit, having used to medicine for $O$. viverrini infection, having an $O$. viverrini infection records, having salad or fermented fish, were significantly associated to $O$. viverrini infection (Thaewnongiew et al., 2014. The figures show that questionnaire with past histories question may useful for screening of $O$. viverrini in the rural areas.

In conclusion, the present study indicated that $O$. viverrini still found to be a problem in rural community of Surin province, especially in the group of age group over 50 year old, education of primary school, and occupation of agriculture. The infection rate was distributed in 11 districts, therefore, mass treatment and health education are urgently required.

\section{Acknowledgements}

This work was supported by Suranaree University of Technology (SUT) and by Office of the Higher Education Commission under NRU Project of Thailand.

\section{References}

Harinasuta C, Vajrasthira S (1960). Opisthorchiasis in Thailand. Am J Trop Med Hyg, 54, 100-5.

Harinasuta T, Riganti M, Bunnag D (1984). Opisthorchis viverrini infection: pathogenesis and clinical features. Arzneimittelforschung, 34, 1167-9.

Jongsuksuntigul P, Imsomboon T (2003). Opisthorchiasis control in Thailand. Acta Trop, 88, 229-32.

Kaewpitoon N, Kaewpitoon SJ, Pengsaa P (2008). Opisthorchiasis in Thailand: review and current status. World J Gastroenterol, 14, 2297-302.

Kaewpitoon SJ, Rujirakul R, Kaewpitoon N (2012). Prevalence of Opisthorchis viverrini infection in Nakhon Ratchasima province, Northeast Thailand. Asian Pac J Cancer Prev, 13, 5245-9.

Kaewpitoon SJ, Rujirakul R, Ueng-Arporn N, et al (2012). Community-based cross-sectional study of carcinogenic human liver fluke in elderly from Surin province, Thailand. Asian Pac J Cancer Prev, 13, 4285-8.

Kato K, Miura M (1954). Comparative examinations. Jpn J Parasitol, 3, 35.

Rujirakul R, Ueng-arporn N, Kaewpitoon S, et al (2015). GISbased spatial statistical analysis of risk areas for liver flukes in Surin Province of Thailand. Asian Pac J Cancer Prev, 
16, 2323-6.

Sadun EH (1995). Studies on Opisthorchis viverrini in Thailand. Am J Hyg, 62, 81-115.

Sithithaworn P, Andrews RH, Nguyen VD, et al (2012). The current status of opisthorchiasis and clonorchiasis in the Mekong Basin. Parasitol International, 61, 10-6.

Sithithaworn P, Pipitgool V, Srisawangwong T, et al., (1997). Seasonal variation of Opisthorchis viverrini infection in cyprinoid fish in north-east Thailand: implications for parasite control and food safety. Bull World Health Organ, 75, 125-31.

Sripa B, Bethony JM, Sithithaworn P, et al., (2011). Opisthorchiasis and Opisthorchis-associated cholangiocarcinoma in Thailand and Laos. Acta Trop, 120, 158-68.

Sripa B, Kaewkes S, Intapan PM, et al (2010). Food-borne trematodiases in Southeast Asia: epidemiology, pathology, clinical manifestation and control. Adv Parasitol, 72, 305-50.

Sripa B, Kaewkes S, Sithithaworn P, et al (2007). Liver fluke induces cholangiocarcinoma. PLoS Med, 4, 201.

Surin Administrative Organization, Thailand. General data of Surin province. Access : http://www.surin.go.th. Retrieved date: 11 September 2013.

Suwannahitatom P, Klomjit S, Naaglor T, et al (2013). A follow-up study of Opisthorchis viverrini infection after the implementation of control program in a rural community, central Thailand. Parasit Vectors, 6, 188.

Thaewnongiew K, Singthong S, Kutchamart S, et al (2014). Prevalence and risk factors for Opisthorchis viverrini infections in upper Northeast Thailand. Asian Pac J Cancer Prev, 15, 6609-12.

Thamavit W, Bhamarapravati N, Sahaphong S, et al (1978). Effects of dimethylnitrosamine on induction of cholangiocarcinoma in Opisthorchis viverrini-infected Syrian golden hamsters. Cancer Res, 38, 4634-9.

Vichasri S, Viyanant V, Upatham ES (1982). Opisthorchis viverrini: intensity and rates of infection in cyprinoid fish from an endemic focus in northeast Thailand. Southeast Asian J Trop Med Public Health, 3, 138-41.

Wykoff DE, Chittayasothorn K, Winn MM (1966). Clinical manifestation of Opisthorchis viverrini infection in Thailand. Am J Trop Med Hyg, 15, 914-8. 